\title{
Factores Relevantes De Competitividad En Pymes
}

\author{
Amadeo Segura Hernández, PhD \\ Universidad Popular Autónoma del Estado de Puebla, México
}

Doi:10.19044/esj.2018.v14n28p193 URL:http://dx.doi.org/10.19044/esj.2018.v14n28p193

\begin{abstract}
Competitiveness in all areas has increased with the opening of trade worldwide. This resulted to greater pressure on entities that have lags compared to others that are more prepared and which are better located internationally. In this context, small and medium-sized enterprises (SMEs) show lower levels of competitiveness than the large industry. This occurs most especially when they are located in developing countries, given the holistic nature of competitiveness. This paper focuses on identifying the relevant factors of competitiveness of SMEs. During the bibliographic research, networks, finances, innovation, and information systems were identified as recurrent preponderant factors in the four levels of systemic competitiveness: micro, meso, macro, and meta. This article shows the result of the first stage of the research, and is structured with concurrent triangulation design to perform cross validation of quantitative and qualitative aspects. The study was performed using a non-probabilistic sampling and with systemic representation through personalized interviews of different people from different groups involved in the system of which the SME's of the State of Puebla, Mexico, are partly related to the automotive and auto parts sector. The four factors were confirmed in this research stage and, at the same time, five other relevant factors were also recognized: knowledge, culture, specialization, diversification, and institutionalization. The identification of the predominant factors of competitiveness offers the possibility of developing them holistically by the various related actors that provide the enabling environment for the strengthening of SMEs.
\end{abstract}

Keywords: Factor of competitiveness, SMEs, Automotive sector

\section{Resumen}

La competitividad en todos los ámbitos se ha incrementado ante la apertura comercial a nivel mundial, esto trae consigo mayor presión a los entes que presentan rezagos comparados contra otros más preparados y por ende mejor ubicados a nivel internacional. En este contexto las pequeñas y medianas empresas (Pymes) muestran niveles inferiores de competitividad que la gran 
industria y en especial cuando están localizadas en países en vías de desarrollo, dado lo holístico de la competitividad. El objetivo de la investigación es identificar los factores relevantes de competitividad de las Pymes. Durante la investigación bibliográfica se identificaron a las redes, finanzas, innovación y los sistemas de información como factores preponderantes recurrentes en los cuatro niveles de competitividad sistémica: micro, meso, macro y meta. En este artículo se muestra el resultado de la primera etapa de la investigación, estructurada con diseño de triangulación concurrente para realizar validación cruzada de aspectos cuantitativos y cualitativos, con muestreo no probabilístico y con representatividad sistémica mediante entrevistas personalizadas a diferentes personas de diversos grupos envueltos en el sistema del que forman parte las Pymes del Estado de Puebla, México, relacionadas con el sector automotriz y de autopartes. Los cuatro factores se confirmaron en esta etapa de investigación y, al mismo tiempo, fueron reconocidos otros cinco factores relevantes: el conocimiento, la cultura, especialización, diversificación e institucionalización. La identificación de los factores preponderantes de competitividad brindan la posibilidad de desarrollarlos holísticamente por los diversos actores relacionados que proporcionen el ambiente propicio para el fortalecimiento de las Pymes.

Palabras clave: factores de competitividad, Pymes, sector automotriz

\section{Introducción}

Según Pro-México (2014), México tiene acuerdos comerciales en tres continentes, con 10 tratados de libre comercio con 45 países, 30 acuerdos para la promoción y protección recíproca de las inversiones y 9 acuerdos de alcance limitado que le abren la puerta a un mercado potencial de mil millones de consumidores y $60 \%$ del Producto Interno Bruto (PIB) mundial. Sin embargo, el intercambio comercial se centra con Estados Unidos de América (EUA) al ser considerado el primer socio comercial por representar el $83 \%$ de las exportaciones según la Secretaría de Economía (SE, 2018).

En esta línea de apertura comercial, la cadena de AutopartesAutomotriz (CAA) a nivel mundial ha sufrido una reestructuración, turnando sus procesos de fabricación hacia Asia, preponderantemente China, desplazando a los europeos y estadounidenses, ya en 2012 la producción en ese país alcanzó la cifra de 19.3 millones de unidades comparada contra los 10.3 millones de Estados Unidos, 2.5 millones de Canadá y los 3 millones de México. Así, los niveles de producción de automóviles por país han fluctuado en los últimos años y se han dado cambios en el ranking de los 40 principales a nivel mundial, según la Organisation Internationale des Constructeurs d'Automobiles (OICA), China adelanto a Estados Unidos y Japón como primer fabricante y como el mercado más grande. 
Según las investigaciones de Chen y Lin (2006), Cho, Moon y Kim (2009) y Hao-Sung (2003), mencionadas por Peña (2010), el crecimiento e industrialización de países asiáticos como: Taiwán, Singapur y Corea es resultado de profesionales cualificados, actitud emprendedora, fuerte competencia local, el buen desempeño de las industrias de tecnología y la integración de redes entre empresarios, centros de estudio y autoridades.

Según Carbajal Suárez (2012), Carrillo y Barajas (2007), citados por Álvarez, Carrillo y González. (2014) entre los principales analistas de CAA, mencionan a este sector como uno de los más dinámicos por su productividad, atracción de Inversión Extranjera Directa (IED), niveles de exportación, portafolio de productos, generación de empleo y balanza comercial positiva. Sin embargo, este sector en México, clasificado en el octavo lugar como fabricante a nivel mundial, aún tiene retos importantes que afrontar, como incrementar su nivel de integración local acorde con las tasas de producción de vehículos que se han alcanzado. El sector aún depende de volúmenes elevados de importaciones para la fabricación completa de un automóvil. La CAA mexicana es una cadena de valor dinámica con bajos niveles de integración del resto del sistema de producción mexicano, carente de ciencia y tecnología, beneficiado por bajos aranceles y costos de traslado.

En este contexto, el sector empresarial en México está compuesto principalmente por Pymes, que representan alrededor del $90 \%$ del número de empresas y su contribución en la generación de empleo es alta, su participación en las exportaciones es baja dado su enfoque primordial en el mercado interno, lo que las hace dependientes de la dinámica de la economía interna (Comisión Económica para América Latina CEPAL, 2011). Entre los grandes desafíos que han afrontado las Pymes, según Ferraro (2011) en un estudio para Naciones Unidas (UN) y para la CEPAL sobre la eliminación de las barreras para el financiamiento de las Pymes en América Latina, se encuentran: la limitada participación en las exportaciones, la escasa vinculación tanto con los sectores dinámicos, así como con las universidades, deficiente capacitación, escasa innovación y limitado acceso a fuentes de financiamiento.

Esta situación es recurrente en el contexto de Latinoamérica, tal como lo muestran estudios de Zevallos (2003), Palomo (2007) y Dussel (2004), que ponen de manifiesto la problemática como niveles bajos de productividad, incompetencia en la gestión y reducidos niveles de capacitación que afrontan las Pymes. En este ambiente, las pequeñas y medianas empresas latinoamericanas han asumido la misma especialización de sus propios países, básicamente caracterizados por enfoque en productos básicos con escaso valor añadido, poca diferenciación y en algunos casos en maquilas de baja tecnología y conocimiento, así mismo, con enfoques defensivos, (Casalet \& González, 2004) no sustentables en el largo plazo, con baja internacionalización, sin integraciones y sin innovaciones, con procesos de altos niveles de mano de 
obra, que permiten absorber la fuerza laboral de estos países, lamentablemente con poca productividad y baja remuneración.

Una alternativa para mejorar la situación de las Pymes que les permitan aprovechar la oportunidad que brinda la CAA en pos de una proveeduría local es lograr niveles de competitividad mejores, dirigiendo esta competitividad desde un enfoque sistémico, tal como lo mencionan Casalet y González (2004), sobre la disrupción del modelo lineal hacia un modelo de redes de cooperación, el cambio en la percepción de lo tangible y la relevancia hacia lo intangible, así mismo, esta autora hace referencia a Boscherini y Poma (2000) y Yoguel (2000) dejando de manifiesto el cambio de las ventajas estáticas hacia una dotación factorial y claramente sistémica, tomando relevancia la competitividad a nivel nacional, regional, en las ciudades, los clústeres y la competitividad empresarial, e inclusive de las personas.

Bajo este contexto holístico surgen cuestionamientos como ¿cuáles son los factores preponderantes de competitividad de las Pymes del sector automotriz y de autopartes? ¿Son estos factores recurrentes en los diferentes niveles determinados por la competitividad sistémica? Para responder a estos cuestionamientos se establecieron 3 objetivos cuantitativos con alcances iniciales tanto exploratorio, como descriptivo, concluyendo en un alcance explicativo. Acotada la investigación a un estado de la República Mexicana, en dónde esta industria es un sector estratégico, unificado de la siguiente manera: explicar los factores actuales que tienen una influencia trascendente en la competitividad sistémica de las Pymes ubicadas en el estado de Puebla del sector automovilístico y autopartes. Como complemento y para lograr un enfoque mixto se definieron 2 objetivos cualitativos: identificar las categorías cualitativas de competitividad sistémica, que permitan, en segunda instancia, especificar y explicar las características de los factores, su estructura y funcionamiento sistémico de las Pymes internamente y en su entorno.

Para alcanzar estos objetivos se revisaron distintos estudios abordados desde diferentes perspectivas, de dónde se obtuvieron diversos factores, que fueron agrupados según la clasificación de competitividad sistémica en los niveles: micro, meso, macro y meta. En este análisis sobresalieron en los cuatro niveles de competitividad sistémica los factores: redes/vinculación, finanzas, innovación y sistemas de información. En el Esquema 1 se muestra un resumen visual de todos los factores identificados y que fueron clasificados en cada nivel. 
Esquema 1. Reconocimiento de Factores de competitividad sistémica.

\begin{tabular}{|c|c|c|c|}
\hline MICRO & MESO & MACRO & META \\
\hline A1 Empresa & \begin{tabular}{|l|l|} 
A2 & Politica \\
\end{tabular} & \begin{tabular}{l|l} 
A3 & Politica/Gobierno
\end{tabular} & \begin{tabular}{l|l} 
A4 & Politica \\
\end{tabular} \\
\hline B1 Empresario & \begin{tabular}{|l|l|} 
B2 & Economía \\
\end{tabular} & \begin{tabular}{|l|l|} 
B3 & Economía \\
\end{tabular} & \begin{tabular}{|l|l|} 
B4 & Economía \\
\end{tabular} \\
\hline \begin{tabular}{|l|l|l} 
C1 & Redes \\
\end{tabular} & \begin{tabular}{|l|l|} 
C2 & Res / Vinculación \\
\end{tabular} & \begin{tabular}{l|l} 
C3 & Redes/Vinculaciór \\
\end{tabular} & \begin{tabular}{|l|l|} 
C4 & Redes \\
\end{tabular} \\
\hline D1 Finanzas & \begin{tabular}{l|l} 
D2 & Financiamiento
\end{tabular} & \begin{tabular}{l|l} 
D3 & Finanzas \\
\end{tabular} & \begin{tabular}{|l|l} 
D4 & Finanzas \\
\end{tabular} \\
\hline \begin{tabular}{|l|l} 
E1 Innovación \\
\end{tabular} & E2 Innovación & \begin{tabular}{|l|l|} 
E3 & Innovación y Tec.
\end{tabular} & \begin{tabular}{|l|l|} 
E4 & Innovación \\
\end{tabular} \\
\hline \begin{tabular}{|l|l} 
F1 & Sist. Inform. (TIC) \\
\end{tabular} & F2 Información & \begin{tabular}{|l|l|} 
F3 & Información \\
\end{tabular} & \begin{tabular}{|l|l|} 
F4 & Información \\
\end{tabular} \\
\hline G1 Estrategía & G2 Infraestructura & \begin{tabular}{|l|l|} 
G3 & Infraestructura \\
\end{tabular} & \begin{tabular}{|l|l|} 
G4 Infraestructura \\
\end{tabular} \\
\hline H1 Gestión & H2 Apoyos & \begin{tabular}{|l|l|} 
H3 & Apoyos \\
\end{tabular} & \begin{tabular}{|l|l|} 
H4 & Apoyos \\
\end{tabular} \\
\hline I1 Organización & 12 Servicios & \begin{tabular}{|l|l|}
13 & Sociedad \\
\end{tabular} & 14 Sociedad \\
\hline \begin{tabular}{|l|l|} 
J1 & Recursos \\
\end{tabular} & J2 Territorio / Región & \begin{tabular}{|l|l|}
$\mathrm{J} 3$ & Territorio \\
\end{tabular} & \\
\hline K1 Procesos & K2 Mercado & \begin{tabular}{l|l} 
K3 & Empresas \\
\end{tabular} & \\
\hline \begin{tabular}{|l|l|} 
L1 & Tecnología \\
\end{tabular} & \begin{tabular}{|l|l|} 
L2 & Tec. inF. y com. (TIC) \\
\end{tabular} & & \\
\hline M1 Valor agregado & & & \\
\hline
\end{tabular}

Elaboración propia (2017)

Al ser estos 4 factores los más recurrentes en la revisión bibliográfica se delimitan a continuación cada uno de ellos, así como, un resumen de los principales autores que los abordaron y se mencionan desde cada una de las perspectivas que fueron estudiados, bajo una concepción holística, donde estos factores son preponderantes tanto en una referencia al interior de las Pymes, en su interactuar directo local-regional, como inclusive en niveles mayores como nacional y global:

\section{Redes/Vinculación}

Conjunto de elementos organizados para determinado fin, una estructura que cuenta con un patrón característico, relacionados para una determinada actividad mediante cooperación, vinculación y/o colaboración. El engrandecimiento de vinculaciones y redes contribuye al desarrollo de las empresas nacionales $\mathrm{y}$, en algunos casos, fomenta la evolución desde una plataforma de ensamblaje hacia un centro de manufacturas e identificación de oportunidades de negocios con empresas transnacionales y cadenas globales de valor. Cuanto más vasta y compleja es la globalización, tanto más articulado es el esquema de gobernanza que se requiere para el desarrollo, expansión y mantenimiento de articulaciones institucionales nacionales con organizaciones extranjeras. Comprende la vinculación entre el sector público y del sistema científico-tecnológico e impacta en el desarrollo socio-económico regional. Comprende redes de cooperación interinstitucional e interempresarial, geográfica y socialmente enraizada, así como, el conocimiento de las cadenas de valor global, los vínculos académico-industriales y los programas de cadenas productivas internacionales. Esta concepción es abstraída de los diversos estudios identificados mencionados en la Tabla 1.

Para Pecina (2011), estos encadenamientos interindustriales e intraindustriales están integrados en redes horizontales y verticales con distintos sectores industriales, enfocados en la rentabilidad de la empresa para reducir la fragilidad de hacerlo de manera aislada. En este contexto, la CEPAL 
y el Fondo Multilateral de Inversión (FOMIN) (2010) realizaron una investigación sobre la articulación productiva, asociatividad y cooperaciones entre Pymes, los resultados demuestran que los resultados más relevantes fueron con respecto a la innovación y la entrada a mercados más competitivos.

\section{Finanzas/financiamiento}

Se refiere al intercambio de distintos bienes de capital entre individuos, empresas, o estados y con la incertidumbre y el riesgo que estas actividades conllevan. Considera la capacidad financiera como por ejemplo la implantación y control de un sistema de contabilidad de costos, presupuesto de ingresos y gastos anuales. También comprende el análisis de la situación económico-financiera. Una opción de medir la competitividad de las empresas a nivel internacional es emplear indicadores financieros tales como ROA (Return on Assets), EVA (Economic value added) y MVA (Market value added) (Chen \& Lin, 2006), sin embargo, este enfoque es limitativo y solo considera indicadores cuantitativos, dejando de lado los aspectos cualitativos (Peña, 2010).

Tabla 1. Redes.

\begin{tabular}{|c|c|}
\hline \multicolumn{2}{|r|}{ REDES/VINCULACIÓN } \\
\hline Variable/factor/Indicador & Autores relacionados \\
\hline Acuerdos de cooperación & $\begin{array}{l}\text { Hoffimann y Schlosser (2001), Klofsten y Scheele (2003), Verhees (2004), } \\
\text { Rubio A. y Aragón A. (2007). }\end{array}$ \\
\hline Articulación institucional & Casalet M. y González L. (2004). \\
\hline Asistencia & Programa AL-INVEST y EUROCHAMBRES, por CEPAL (2012). \\
\hline Asociaciones-clústeres & Brunet I. y Santamaría C. (2012). \\
\hline Cadenas globales de valor & $\begin{array}{l}\text { Cuevas H., Rangel J. y Hernández O. (2014), Dini M. y Stumpo G (2011), } \\
\text { Martin D. y Tinoco M. (2010), Banco Mundial (2006). }\end{array}$ \\
\hline Capacidad de relacionarse & Dini M. y Stumpo G (2011). \\
\hline Capacidades colectivas & Brunet I. y Santamaría C. (2012). \\
\hline Cooperación comercial e interempresarial & $\begin{array}{l}\text { Minguez R. (2010), Camisón (1999), Rubio A. y Aragón A. (2007), Verhees } \\
\text { (2004). }\end{array}$ \\
\hline Cooperación productiva y tecnológica & Mínguez R. (2010). \\
\hline $\begin{array}{l}\text { Empresarialidad, gestión y cooperación } \\
\text { empresarial }\end{array}$ & Montoya L. Montoya I. y Castellanos O. (2008). \\
\hline Encadenamientos productivos & $\begin{array}{l}\text { CEPAL, el Programa AL-INVEST y EUROCHAMBRES (2012), Ferraro C. } \\
\text { (2011), Lecuona (2009). }\end{array}$ \\
\hline Eslabonamientos & Casalet M. y González L. (2004). \\
\hline Esquemas asociativos & Alarcón y Stumpo (2004). \\
\hline $\begin{array}{l}\text { ortalecimiento de las cadenas productivas } \\
\text { y las redes de empresas }\end{array}$ & Saavedra, M. L. y Tapia B. (2011), Listerri (2002). \\
\hline Integraciones & $\begin{array}{l}\text { Montoya L. Montoya I. y Castellanos O. (2008), Guerrieri, Pietrobelli (2003), } \\
\text { Cerdan (2005). }\end{array}$ \\
\hline Organización de intereses locales & Brunet I. y Santamaría C. (2012). \\
\hline Proyectos de Integración Productiva (PIP) & Dini M. y Stumpo G (2011). \\
\hline Redes & Rialp A., Rialp J. y Knight G. (2010), Coviello y Cox (2006). \\
\hline Redes empresariales & Brunet I. y Santamaría C. (2012), ONUDI (2002 y 2005), PNUD (2000). \\
\hline Redes horizontales y verticales & Dini M. y Stumpo G (2011), ONUDI (2005) y López (2003). \\
\hline $\begin{array}{c}\text { pistemas productivos en conglomerados o } \\
\text { clústeres }\end{array}$ & Villarreal A. (2010). \\
\hline Vinculación y asistencia & $\begin{array}{l}\text { CEPAL, el Programa AL-INVEST y EUROCHAMBRES (2012), Dussel E. } \\
\text { (2004). Martin D. y Tinoco M. (2010), Banco Mundial (2006). }\end{array}$ \\
\hline
\end{tabular}

Elaboración propia (2017)

Así mismo, la configuración del sistema financiero influye decisivamente en el grado de acceso al financiamiento de las Pymes. En el aspecto meta, se debe 
fomentar el uso de instrumentos específicos para facilitar el acceso a bienes de capital globales, para aportar en el acceso al crédito y al financiamiento, se debe brindar un paquete de servicios financieros. Los países deben generar e implantar programas para su competitividad considerando como objetivo el aumento de la productividad de los factores de la producción, sustentados por medio del desarrollo de mercados financieros, con bancos de desarrollo, siendo trascendente la sofisticación del mercado financiero. En este panorama uno de los principales problemas al que se enfrentan las Pymes y el emprendimiento son el acceso y las condiciones para el financiamiento, con todas sus limitaciones. Esta delimitación fue obtenida de las diferentes referencias identificadas mencionadas en la Tabla 2.

Una muestra de ello es el estudio realizado por la CEPAL (2011) y la Agencia Española de Cooperación Internacional para el Desarrollo (AECID) a 600 Pymes ubicadas en tres países de Centroamérica que muestra que el 57\% financia sus actividades con sus propios recursos y hasta un 75\% los empleó para iniciar operaciones. Aquellas que logran acceder al crédito son generalmente bancarias y de corto plazo. Esto restringe la creación de nuevas empresas, dificulta la ampliación de las actuales y en otros casos complica la operación normal (CEPAL, 2011).

Tabla 2. Finanzas.

\begin{tabular}{|c|l|}
\hline \multicolumn{2}{|c|}{ FINANZAS / FINANCIAMIENTO } \\
\hline $\begin{array}{c}\text { Variable/factor/Indicador } \\
\text { mercados de factores y bienes }\end{array}$ & Montores relacionados \\
\hline Acceso al crédito y al financiamiento & $\begin{array}{l}\text { Ferraro C. (2011), Milesi D., Moori V., Robert V. y Yoguel G. (2007), Berry } \\
\text { Albert (2002). }\end{array}$ \\
\hline Apalancamiento & Dussel E. (2004), Garrido (2002). \\
\hline Área financiera & Saavedra, M. (2012). \\
\hline Banca de desarrollo & Ferraro C. (2011). \\
\hline Capacidades financieras & Aragón A. y Rubio A. (2005). \\
\hline Contabilidad y finanzas & Saavedra, M. (2012), Martínez y Álvarez (2006), Zeballos (2001). \\
\hline $\begin{array}{c}\text { Desarrollo y disponibilidad de mercados } \\
\text { financieros }\end{array}$ & $\begin{array}{l}\text { Listerri (2002), Hausmann, Ricardo, Lozoya, Emilio y Mia, Irene (2009), WEF } \\
\text { (2009). }\end{array}$ \\
\hline Factores financieros & Martinez J. (2007). \\
\hline $\begin{array}{c}\text { Financiamiento y tasas de interés } \\
\text { (2006), Montoya L. Montoya I. y Castellanos O. (2008), Botero (2005), BID } \\
\text { (2001). }\end{array}$ \\
\hline Mercado de deuda para Empresas & Flores V. y Vargas J. (2013), Observatorio Pyme (2013). \\
\hline Mercado financiero & Brunet I. y Santamaría C. (2012). \\
\hline $\begin{array}{c}\text { Participaciones más elevadas de los } \\
\text { pequeños bancos }\end{array}$ & Ferraro C. (2011), Ascúa (2009). \\
\hline Reducción de costos & CEPAL, el Programa AL-INVEST y EUROCHAMBRES (2012). \\
\hline Sistema y servicios Financieros & Ferraro C. (2011). \\
\hline Sofisticación del mercado financiero & Hausmann, Ricardo, Lozoya, Emilio y Mia, Irene (2009), WEF (2009). \\
\hline Solvencia & Rubio A. y Aragón A. (2007). \\
\hline
\end{tabular}

Elaboración propia (2017)

\section{Innovación}

Es un cambio que introduce novedades, es un proceso que consiste en convertir en una solución a un problema o una necesidad. No constituye una 
serie de eventos aislados o puntuales, sino un proceso acumulativo o trayectoria de acciones. Es relevante el enfoque de la dirección en este sentido y como se permea a toda la organización. Es un potencial para generar innovación endógena, es una estrategia clave de la competitividad nacional en el largo plazo, donde la innovación intersectorial es crucial en la nueva visión del desarrollo regional como parte integral del entorno doméstico, bajo una perspectiva de meso-macro economía. Comprenden mejoras y ajustes estructurales, así como, organizacionales a partir de implementación y/o mejora de procesos de administración. Es aplicable también a productos y procesos. Incluye la interacción de la empresa con el entorno productivo, institucional para la generación de innovaciones y como todo lo anterior se refleja en la capacidad de generar innovaciones en sucesión rápida.

Los procesos complejos de innovación, tanto a nivel nacional, como regional y local, en la generación, distribución y aplicación de innovaciones requiere de mecanismos de gobernanza más articulados, en el que el sector público desempeña una función proactiva muy importante. Estos conceptos fueron identificados de diversas fuentes reconocidas y enunciadas en la Tabla 3.

Otro factor que influye en la competitividad corresponde a los sectores conectados y de soporte, que comprende principalmente a los proveedores, en tanto mejores niveles tengan de innovación, competitividad y presencia internacional se incrementa el nivel de la empresa misma en competitividad internacional. De igual manera sectores competitivos incitan el desarrollo de nuevas divisiones y la conservación de ventajas competitivas alcanzadas (Jin \& Moon, 2006).

Tabla 3. Innovación.

\begin{tabular}{|c|l|}
\hline \multicolumn{2}{|c|}{ INNOVACIÓN } \\
\hline Variable/factor/Indicador & \multicolumn{1}{|c|}{ Autores relacionados } \\
\hline $\begin{array}{c}\text { Apoyo a la innovación y difusión } \\
\text { tecnológica }\end{array}$ & Listerri (2002). \\
\hline Capacidad innovadora & Camisón, Lapiedra, Segarra, y Boronat (2004). \\
\hline Innovación & $\begin{array}{c}\text { Aragón A. y Rubio A. (2005), Ribeiro (2003), Piñero J., Berrocal F. y Edwards } \\
\text { J. (2013), Coduras A. (2010), Hausmann, Ricardo, Lozoya, Emilio y Mia, Irene } \\
\text { (2009), WEF (2009), Díaz Barriga A. (2003), Flores B. y González F. (2009). }\end{array}$ \\
\hline $\begin{array}{c}\text { Innovaciones de producto, proceso, } \\
\text { marketing y distribución }\end{array}$ & $\begin{array}{l}\text { Dini M. y Stumpo G (2011), Rodríguez (2013). CEPAL, el Programa AL-INVEST } \\
\text { y EUROCHAMBRES (2012), Rubio A. y Aragón A. (2007), Camisón, Lapiedra- } \\
\text { Alcamí, Segarraciprés y Bornat-Naverro (2004). }\end{array}$ \\
\hline $\begin{array}{c}\text { Innovaciones organizacionales } \\
\text { Número de investigadores y patentes }\end{array}$ & Rodríguez (2013), OCDE (2005). \\
\hline Pre-innovación & Dini M. y Stumpo G (2011), Aggio y Milesi (2008). \\
\hline Sistema de innovación & Dini M. y Stumpo G (2011), Aggio y Milesi (2008) \\
\hline Sistemas regionales de innovación (SRI) & Brunet I. y Santamaría C. (2012), De la Mothe y Paquet (2008). \\
\hline Tecnología e innovación & Saavedra, M. (2012), Zevallos (2007). \\
\hline
\end{tabular}

Elaboración propia (2017)

En este sentido, según el reporte del WEF (2013-14), en el futuro cercano la capacidad de innovación en productos, procesos y modelos de negocio que tengan las economías será un factor determinante, para ello los 
sistemas educativos desempeñarán un rol trascendente tanto para la actividad empresarial, como en el desarrollo de la capacidad de innovación y el emprendimiento. En este entorno, el Banco interamericano de Desarrollo, Fundes y un grupo de investigadores multidisciplinarios, realizaron en 2008 un estudio en un número mayor de 400 Pymes localizadas en Argentina, Colombia, Costa Rica y Chile, para analizar la relación entre la innovación, el comportamiento de las exportaciones y el impacto social. Los resultados mostraron que existe correspondencia positiva entre la innovación y el éxito exportador, a mayor énfasis en la innovación mejores resultados cuantitativos y cualitativos.

\section{Sistemas de información}

Es el conjunto de tecnologías desarrolladas para gestionar información y enviarla de un lugar a otro. Incluyen las tecnologías para almacenar información y recuperarla después, enviar y recibir información de un sitio a otro, o procesar información para poder calcular resultados y elaborar informes. Es un conjunto organizado de datos procesados, que constituyen un mensaje que cambia el estado de conocimiento del sujeto o sistema que recibe dicho mensaje, esto permite a las empresas, organizaciones, gobiernos o sistemas que poseen dicho estado nuevo de conocimiento tomar decisiones pertinentes acordes a dicho conocimiento.

Así mismo, considera la fiabilidad, seguridad, facilidad de uso, la eficacia y eficiencia para los fines previstos, combinan correctamente los conocimientos, prácticas y experiencias para atender tanto la infraestructura como la tecnología de información de una organización y las personas que lo utilizan. Permite la interacción con la realidad de una manera más rápida y se busca que sea a un costo marginal. Respecto a la injerencia de las instituciones, asociaciones y entes empresariales en la radiación de competitividad, procesos en los países desarrollados funcionan actualmente de manera autónoma, en tanto que en los países en vías de desarrollo en primera instancia se deben crear, en otros tantos impulsarlos. En la Tabla 4 se enlistas diferentes autores con sus respectivas variables y/o indicadores que han abordado los sistemas de información y su relación con la competitividad.

Tabla 4. Sistemas de Información.

\begin{tabular}{|c|l|}
\hline \multicolumn{2}{|c|}{ SISTEMAS DE INFORMACIÓN } \\
\hline Variable/factor/Indicador & \multicolumn{1}{c|}{ Autores relacionados } \\
\hline Acceso a información & Hausmann, Ricardo, Lozoya, Emilio y Mia, Irene (2009), WEF (2009). \\
\hline $\begin{array}{c}\text { Disponibilidad, comparabilidad y } \\
\text { confiabilidad de la información }\end{array}$ & CEPAL, el Programa AL-INVEST y EUROCHAMBRES (2012). \\
\hline Información de mercados & CEPAL, el Programa AL-INVEST y EUROCHAMBRES (2012). \\
\hline Información sobre Pymes & Guaipatín, C. (2003). \\
\hline Sistemas de información & Saavedra M. (2012), RICYT (2009), Martínez y Álvarez (2006). \\
\hline $\begin{array}{c}\text { Tecnologías de la Información y } \\
\text { Comunicación (TIC) }\end{array}$ & $\begin{array}{l}\text { Casalet M. y González L. (2004), Stiglitz (2003), Rialp A., Rialp J. ynght G. } \\
\text { (2010). }\end{array}$ \\
\hline
\end{tabular}

Elaboración propia (2017) 
Para la contrastación del marco teórico contra la realidad del sistema del que forman parte las Pymes de los sectores automovilístico y autopartista de Puebla, con base en la información obtenida se establece la hipótesis de investigación: Los factores preponderantes causales de competitividad sistémica de las Pymes de los sectores automovilístico y autopartista de Puebla son las redes/vinculación, las finanzas, la innovación y sistemas de información. Compet. Sistém. Fact. Prepond. Causales f Redes-Vinculación + Finanzas + Innovación + Información.

\section{Materiales y Métodos}

Para enriquecimiento de la investigación concerniente a los factores preponderantes de competitividad sistémica, esta se aborda desde los dos extremos para una conclusión central, mediante el Diseño de triangulación concurrente (DITRIAC), el concepto de la investigación completa se muestra en el esquema 2, este diseño mixto nos permite corroborar resultados y realizar validación cruzada entre datos cuantitativos y cualitativos, capitalizando las ventajas de cada uno y reducir sus desventajas. El enfoque cuantitativo puede llegar a generar leyes y teorías, y el cualitativo nos permite conceptualizar los datos a las generalizaciones, no estadísticas, y suscitar teorías (Hernández Sampieri, 2014). Con lo que se ambiciona mayor robustez científica al considerar ambos métodos de manera integral con la metodología multimétodos. El enfoque mixto nos permite abordar la lógica deductiva, de lo general a lo particular, nombrado análisis por la disgregación de las partes para su profundización y conocer su composición, característico del enfoque cuantitativo, y a su vez suplementarlo con el enfoque cualitativo, con su lógica inductiva, de lo particular a lo general, llamado síntesis por la obtención de un todo a partir de sus partes. Esta forma de abordarlo holísticamente está alineado por la necesidad de concatenar tanto el análisis, como la síntesis por la competitividad sistémica, base de la pesquisa.

Para contrastar la hipótesis de investigación, en este primer alcance de la investigación y que compete a este artículo, se empleará el muestreo no probabilístico, la selección de los entes participantes tiene como principal objetivo integrar a las diferentes poblaciones relacionadas con el sistema poblano de ambos sectores, automotriz y autopartes, en el que intervienen las Pymes y no por un criterio estadístico de representatividad sobre sus respectivas poblaciones, entre los que se encuentran: propietarios de Pymes, gerentes encargados de la operación del negocio, colaboradores de Pymes, así como ciertos proveedores y clientes, expertos en competitividad, profesores de universidades de Puebla, investigadores locales, empleados del gobierno estatal de Puebla y del municipio, representantes del gobierno federal y finalmente integrantes de organismos empresariales. Partiendo de la 
delimitación de Pymes por la cantidad de personal que la compone, en este caso asciende como valor oficial en México a 250 empleados.

Se optó por un estudio de pilotaje no estadístico por lo cerrado del sistema y lo complejo de la participación de las personas en los procesos de investigación, así mismo, la amplitud de los grupos considerados incrementa grandemente la población a muestrear, que lo vuelve difícil de implementar, sobre todo por la selección, en esta fase del estudio, de recolección de información mediante entrevistas personalizadas, como mejor herramienta identificada y probada en la aplicación, porque permite la confianza, disponibilidad y apertura de las personas a contestar.

Como este método no nos permite estimar con exactitud el nivel de confianza la información recabada de estas muestras será empleada en los análisis exploratorios y descriptivos, dado que en estos diseños no se busca representatividad, más bien una escrupulosa y controlada selección de entes con conocimiento amplio del tema en estudio.

Esquema 2. Diseño de investigación

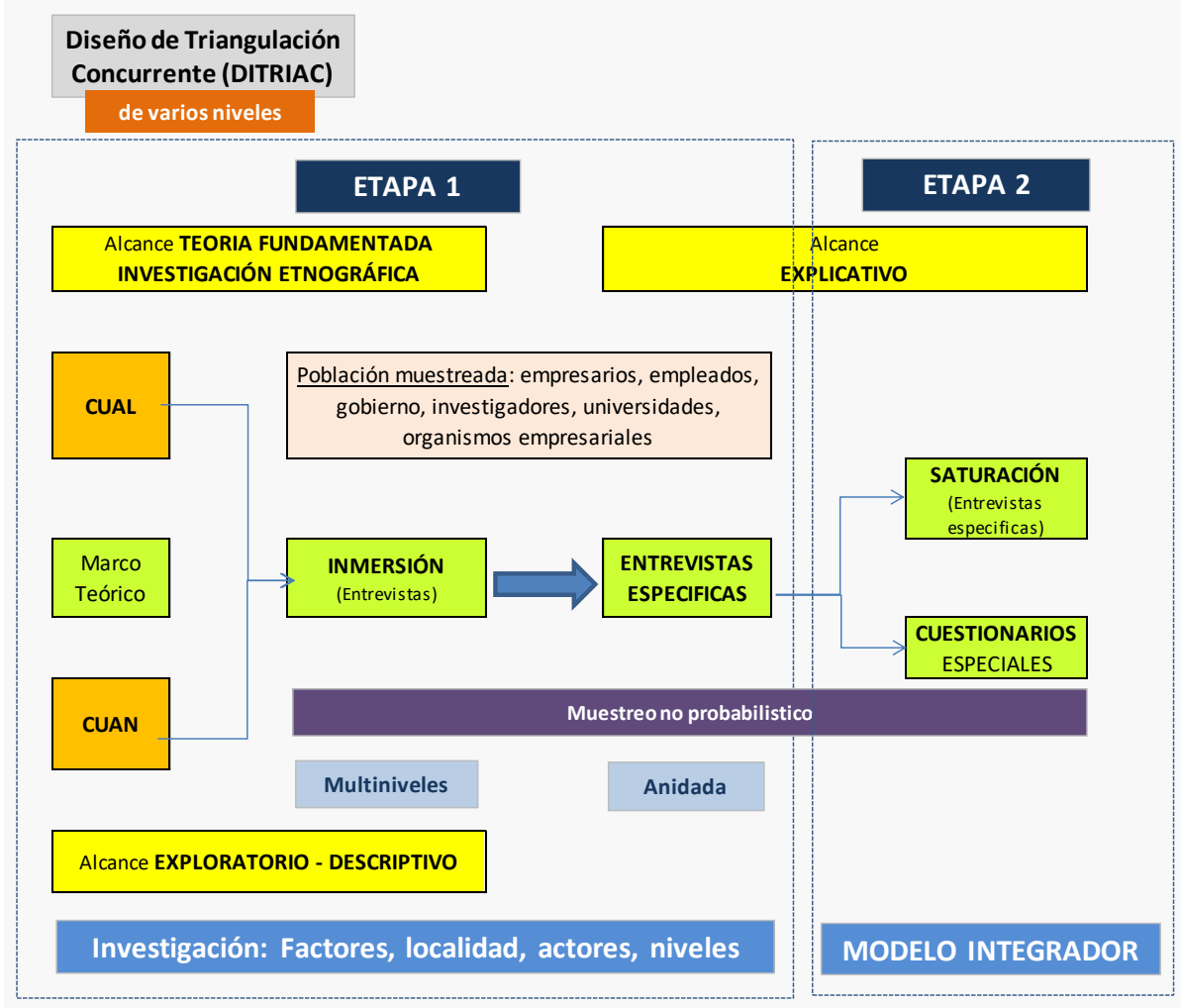

Elaboración propia (2017)

\section{Resultados}

En el Marco Teórico se identificaron los siguientes factores relevantes según distintas acepciones de variadores autores: Redes/Vinculación, 
Finanzas/financiamiento, Innovación, Sistemas de información, Estrategia, Gestión, Organización, Recursos, Procesos, Tecnología, Valor agregado, Política, Economía, Infraestructura, Apoyos, Servicios, Mercado, Bienestar, Emprendedurismo y Nodos. Estos factores fueron resumidos y concentrados con los diversos niveles de la competitividad sistémica, incluyendo locaciones y entes, dónde sobresalieron, como comentado previamente, las Redes/Vinculación, finanzas/financiamiento, la innovación y los sistemas de información, porque eran reiterativas en la inclusión en todos los niveles, otros cuatro factores fueron recurrentes en tres niveles a saber política, economía, infraestructura y los programas de apoyo. Los primeros 4 factores fueron revisados durante el proceso de la etapa 1 de la pesquisa y ratificados como factores relevantes. Así mismo, durante las entrevistas se reconoció que ciertos conceptos que habían sido considerados previamente no correspondían propiamente a factores, en cambio fueron considerados en la división de entes por ejemplo empresa, empresario, gobierno y sociedad, así mismo, los territorios y regiones fueron incorporados en la división de locación, ambas agrupaciones fueron base para otra investigación complementaria, por lo cual han sido abordados en otros artículos con un tratamiento específico.

Durante esta fase se identificaron nuevos factores no detectados durante la revisión del Marco Teórico, entre los que se encuentran: comunicación, creencia en el emprendimiento, cultura, disciplina, especialización, diversificación, orientación al cliente, flexibilidad, institucionalización y resilencia, también se volvió a identificar en este proceso un concepto relacionado con la localización, nombrado como ubicación territorial. Al mismo tiempo, fueron descartados los factores enlistados en el marco teórico como servicios, bienestar y emprendedurismo, porque no fueron confirmados durante este proceso de inmersión y de entrevistas específicas.

Con respecto a los nuevos factores se identificaron un grupo de ellos que corresponden al interior de las Pymes y algunos de ellos en sus relaciones directas con el exterior, básicamente haciendo referencia al nivel micro, entre los que se encuentran: comunicación, creencia en el emprendimiento, orientación al cliente, disciplina, flexibilidad y resilencia. En cambio, el segundo bloque abarca más de dos de los cuatro niveles de competitividad sistémica micro meso, macro y meta, corresponden al: conocimiento, cultura, especialización, diversificación e institucionalización.

\section{Conclusión}

El resultado de la investigación, que ha estado sustentado por el marco teórico de diversos autores y la recolección directa de información con entrevistas con distintas personalizadas involucradas en el sistema del que forman parte las Pymes poblanas de los sectores automotriz y autopartes durante el proceso de inmersión y con entrevistas específicas, ambas bajo un 
muestreo no probabilístico, muestran que los principales factores identificados como preponderantes para lograr la competitividad son aquellas que están enraizadas en los cuatro niveles sistémicos. De manera inicial solo habían sido identificadas las redes/vinculación, finanzas/financiamiento, innovación y sistemas de información, como conclusión a esta primera etapa de investigación se han incorporado el conocimiento, la cultura, especialización, diversificación e institucionalización.

Estos factores son holísticos, están insertados dentro del sistema del que crean y forman parte las Pymes, son intrínsecos con los actores y organizaciones envueltos, así mismo, se relacionan, desarrollan y comportan en concordancia con los niveles de competitividad sistémica que más preponderancia tengan en el sistema mismo, el entorno de la localidad y la relación de esta con el resto de las zonas aledañas o con las que está vinculada.

En la segunda etapa de la investigación se buscará la saturación mediante cuestionarios especiales y entrevistas específicas con focalización en estos nueve factores. Los resultados serán reportados en un artículo subsecuente. Las otras vistas de competitividad identificadas como localidad, actores y organizaciones relevantes, así como, los niveles de competitividad sistémica son reportados en otros artículos complementarios.

Respecto a investigaciones futuras, se recomienda hacer un estudio tomando otro sector como grupo de control, con un diseño de investigación enfocado a buscar la correlación de estos nueve factores. Para el estado de Puebla se vislumbra la opción de llevar a efecto un análisis contra otra industria, se propone dirigirla hacia la industria hospitalaria que ha logrado inversiones nuevas y el crecimiento en su infraestructura en los últimos años. Al mismo tiempo, permitirá contrastar entre un sector industrial y uno de servicios.

\section{References:}

1. Aggio, C. \& Milesi, D. (2008). "Éxito exportador, innovación e impacto social: un estudio exploratorio de PYME exportadoras latinoamericanas". Fundes y el BID (a través del Fondo Fiduciario para el Comercio y la Reducción de la Pobreza).

2. Alarcón, C. \& Giovanni, S. (2004). Articulación productiva en el subsector vitivinícola chileno: el caso de la asociación de productores de vinos finos de exportación (CHILEVID, A.G.). Pequeñas y medianas empresas y eficiencia colectiva. Estudios de caso en América Latina. México, Siglo XXI, Naciones Unidas, pp.139-182.

3. Álvarez, L., Carrillo, J. \& González, M.L. (2014). El auge de la industria automotriz en México en el siglo XXI. Reestructuración y Catching up. Publicaciones empresariales UNAM FCA Publishing, México. 
4. Aragón, A. \& Rubio, A. (2005). Factores explicativos del éxito competitivo: el caso de las Pymes del estado de Veracruz. Universidad de Murcia, España.

5. Arroyo, B., Espinosa, R. \& Erazo, F. (2011). Diagnóstico de las MIPYME del sector industrial en el estado de Guanajuato, México y su visión de negocios." En las memorias del XXI Congreso Latinoamericano de espíritu empresarial. Universidad ICESI, Colombia.

6. Ascúa, R. (2009). La importancia del proceso emprendedor en la Argentina post crisis 2002 y las asimetrías en la evaluación de factores influyentes en el financiamiento de empresas jóvenes. Repositorio Digital CEPAL. Documentos de Proyectos No. 41 pp. 63.

7. Banco, M. (2006). Informe sobre el desarrollo mundial. Equidad y desarrollo. Un mejor clima de inversión para todos. Una coedición del Banco Mundial, Mundi-Prensa y Mayol Ediciones, S.A.

8. Berry, A. (2002). Valoración de políticas de apoyo a la pequeña empresa: Primera aproximación a una metodología regional. Banco interamericano de desarrollo. Departamento de desarrollo sostenible.

9. BID - Banco Interamericano de Desarrollo (2001). Guía operativa para programas de competitividad para la pequeña y mediana empresa. Serie de buenas prácticas del Departamento de Desarrollo Sostenible, 1-31.

10. Boscherini, F. \& Poma, L. (2000). Territorio, conocimiento y competitividad de las empresas. El rol de las instituciones en el espacio global. Niño y Dávila Editores.

11. Botero, J. (2005). Problemas de competitividad para el crecimiento de largo plazo. Presentación Ministerio de Comercio, industria y turismo. Septiembre 13 de 2005.

12. Brunet, I. \& Santamaría, C. (2012). Desarrollo regional y Pymes innovadoras. Anuario electrónico de Estudios en comunicación social Disertaciones, 5 (2). Artículo 4.

13. Camisón, C., Lapiedra, R., Segarra, M. \& Boronat, M. (2004). A metaanalysis of innovation and organizational size, Organization Studies, 25(3), pp. 331-361.

14. Carbajal, Y. (2012). El sector automotriz en el Estado de México. Condiciones y retos de la cadena productiva. Dialnet, Paradigma económico, ISSN 2007-3067, Año 4, N. 2, 2012, págs. 29-59.

15. Carrillo, J. \& Barajas, R. (2007). Maquiladoras fronterizas. Evolución y heterogeneidad en los sectores electrónico y automotriz. México, Miguel Ángel Porrúa/El Colef.

16. Casalet, M. \& González, L. (2004). Las tecnologías de la información en pequeñas y medianas empresas mexicanas. Universidad de Barcelona Vol. VIII, núm. 170. 
17. CEPAL, el Programa AL-INVEST y EUROCHAMBRES (2012). Como mejorar la competitividad de las Pymes en la Unión Europea y América Latina y el Caribe. Naciones Unidas. Impreso en Santiago, Chile.

18. CEPAL (2011). Panorama social de América Latina. Publicación de las Naciones Unidas. ISBN: 978-92-1-221087-2

19. Cerdan (2005). Lecciones aprendidas sobre Cadenas productivas como estrategias de desarrollo. Medellin.

20. Coduras, A. (2010). Capacidades Tecnológicas e innovadoras en las Pymes. Para la competencia en mercados globales. Instituto de Empresa, Business School.

21. Coviello, N.E. \& Cox, M. (2006). The resource dynamics of international new venture networks. Journal of International Entrepreneurship, 4(2-3), 113-132.

22. Cuevas, J., Rangel, J. \& Hernández, O. (2014). La influencia de las actividades de innovación y la gestión del conocimiento en la competitividad de las Pymes manufactureras - un estudio empírico. Ecorfan. Desarrollo Económico en el crecimiento empresarial 53 68.

23. De la Mothe, J. \& Paquet, G. (2008). Local and regional systems of innovation. Kluwer, Londres.

24. Dini, M. \& Stumpo, G. (2011). Políticas para la innovación en las pequeñas y medianas empresas en América Latina. Naciones Unidas y CEPAL.

25. Dussel, E. (2004). Pequeña y mediana empresa en México: condiciones, relevancia en la economía y retos de política. División de estudios de posgrado de la facultad de economía de la UNAM.

26. Ferraro, C. (2011). Eliminando barreras: El financiamiento a las Pymes en América Latina. Naciones Unidas \& Cepal.

27. Flores, V. \& Vargas, J. (2013). Gobierno corporativo en las Pymes mexicanas, una estrategia competitiva. Ecorfan. Congreso Interdisciplinario de cuerpos académicos. Pág. 86 - 102.

28. Flores, B. \& González, F. (2009). La competitividad de las Pymes morelianas. Cuadernos del Cimbage No. 11. Págs. 85 - 104.

29. Fondo Multilateral de Inversión (FOMIN) (2010). Ampliando el acceso a financiamiento, mercados, capacidades y servicios básicos en América Latina y el Caribe.

30. Garrido, C. (2011). Nuevas políticas e instrumentos para el financiamiento de las PYMES en México durante la última década. Oportunidades y desafíos. CEPAL.

31. Guaipatín, C. (2003). Observatorio Mypime: compilación estadística para 12 países de la región. Banco interamericano de desarrollo. 
32. Guerrieri, P. \& Pietrobelli, C. (2003). Industrial districts' evolution and technological regimes: Italy and Taiwan. Universita' di Roma 'La Sapienza', Rome, Italy, Technovation XX.

33. Hao-Sung, J. (2003). The role of information Technology in international competitiveness: Taiwan's experience. Dissertation, Library and archive Canada.

34. Hausmann, R., Lozoya, E. \& Mia, I. (2009). The Mexico competitiveness Report 2009. World Economic Forum. Harvard University.

35. Hernández Sampieri, R. (2014). Metodología de la investigación. México: Mc Graw-Hill.

36. Hoffmann, W. H. \& Schlosser, R. (2001). Success factors of strategic alliances in small and medium-sized enterprises an empirical survey. Long Range Planning, 34(3), pp. 357-362.

37. Klofsten, M. \& Scheele, J. (2003). Innovation and small enterprises in the Third World. International Small Business Journal, 21(3), 354-357.

38. Lecuona Valenzuela, R. (2009). El financiamiento a las Pymes en México, 2000-2007: el papel de la banca de desarrollo, Serie Financiamiento del Desarrollo, CEPAL, Santiago de Chile.

39. Listerri, J.J. (2002). Competitividad en el territorio y desarrollo económico y local. El mercado de valores, año LXII, núms. 3 y 4 , Nacional Financiera. México, D.F., marzo-abril, pp. 3-13.

40. Martin, D. \& Tinoco, M. (2010). El clúster de servicios educativos en Puebla: motor económico. Revista EAN No. 68. Bogotá, Pp. 42-55.

41. Martínez, J. \& Álvarez, C. (2006). Mapa de Competitividad para el diagnóstico de PYMES. En las memorias XI Foro de Investigación. Congreso Internacional de Contaduría, Administración e Informática. México, D.F., octubre.

42. Martinez, J. (2007). Factores que inciden en el desempeño exportador de las Pymes: Una aplicación empírica. Tec. Empresarial. Vol. 1 Ed. 4.

43. Milesi, D., Moori, V., Robert, V. \& Yoguel, G. (2007). Desarrollo de ventajas competitivas: Pymes exportadoras exitosas en Argentina, Chile y Colombia. Revista de la CEPAL 92.

44. Mínguez, R. (2010). Cooperación para la internacionalización como estrategia de expansión exterior de la Pyme. Dirección de análisis y Estrategia. Consejo superior de cámaras de comercio, industria y navegación.

45. Montoya, L.A., Montoya, I.A. \& Castellanos, O.F. (2008). De la noción de competitividad a las ventajas de la integración empresarial. Universidad nacional de Colombia. 59 - 70. 
46. Observatorio Pyme (2013-2014). Evolución reciente, situación actual y desafíos para 2015. Tema especial: El entorno local y el acceso a infraestructura de las Pymes industriales. ISBN 978-987-1659-22-7

47. Palomo, M. (2007). La gestión de procesos y el desempeño competitivo de las PYMES. Revista Ingenierías, 10 (35), 36- 41.

48. Pecina, M. (2011). Clusters y competitividad. Eumed.

49. Peña, V. \& Jesús, C. (2010). Tesis doctoral: Factores determinantes de la competitividad internacional: Aplicación empírica en países en vías de desarrollo (2010). Universidad de Sevilla. Facultad de ciencias económicas y empresariales. Departamento de administración de empresas y marketing. Sevilla, España, Piñero, Juan Enrique, Berrocal, Francisca \& Edwards, José Tomás (2013). Creando oportunidades de innovación y competitividad en las empresas. El proyecto In.Pyme.

50. ProMéxico (2012). Industria Terminal Automotriz. Unidad de Inteligencia de Negocios 2012.

51. Rialp, A., Rialp, J. \& Knight, G. (2010). La vocación global de los nuevos modelos Pymes. El caso de las empresas Born Globals. Cases Study.

52. Ribeiro, D. (2003). Rendimiento de las Pymes innovadoras. Revista Europea de Dirección y Economía de la Empresa, vol. 12, núm. 3, pp. 119-132.

53. Rojas, E., Cuadrado-Roura, J. \& Fernández, J.M. (2005). Gobernar las metrópolis. Banco Interamericano de desarrollo. Washington, D. C.

54. Rubio, A. \& Aragón, A. (2007). Recursos estratégicos en las Pymes. Universidad de Murcia. Revista Europea de Dirección y Economía de la Empresa, vol. 17, núm. 1. pp 103 - 126.

55. Saavedra, M. L. \& Tapia, B. (2011). Mejores prácticas y factores de competitividad en las micro, pequeñas y medianas empresas mexicanas. Universidad de los Andes Venezuela. Redalyc.org. pp. 11 -36 .

56. Saavedra, M.L. (2012). Una propuesta para la determinación de la competitividad en la Pyme latinoamericana. Universidad del norte, Barranquilla, Colombia. Redalyc.org. PP. 93 - 124.

57. Secretaría de Economía (SE, 2018)

58. Stiglitz, J. (2003). Hacia una economía sustentable: conflicto y postconflicto en Colombia. Conferencia dictada en Bogotá, Marco 9 de 2003.

59. Verhess, F. J. \& Meulenberg, M. T. (2004). Market Orientation, Innovativeness, Product Innovation, and Performance in Small Firms. Journal of Small Business Management, vol. 42, núm. 2, pp. 86-91.

60. Villarreal, A. (2010). Cadenas productivas de alto valor/Clusters de México. Instituto Tecnológico de Monterrey. 
61. World Economic Forum WEF (2009). The Global Competitiveness Report $2009-2010$.

62. Yoguel (2000). Sistemas locales de innovación y el desarrollo de la capacidad innovativa de las firmas: las evidencias del cuasi distrito industrial de Rafaela. Redalyc. ISSN: 0328-3186

63. Zevallos, E. (2007). Restricciones del entorno a la competitividad empresarial en América Latina. San José, Costa Rica: FUNDES. 